\title{
Cavity-enhanced single photons from a quantum dot
}

\author{
Jelena Vučković, David Fattal, Dirk Englund, Edo Waks, Charles Santori, Glenn Solomon, and \\ Yoshihisa Yamamoto \\ Ginzton Laboratory, Stanford University, Stanford, CA 94305-4088, U.S.A.
}

\begin{abstract}
Single-photon sources rarely emit two or more photons in the same pulse, compared to a Poisson-distributed source of the same intensity, and have numerous applications in quantum information science. The quality of such a source is evaluated based on three criteria: high efficiency, small multi-photon probability, and quantum indistinguishability. We have demonstrated a single-photon source based on a quantum dot in a micropost microcavity that exhibits a large Purcell factor together with a small multi-photon probability. For a quantum dot on resonance with the cavity, the spontaneous emission rate has been increased by a factor of five, while the probability to emit two or more photons in the same pulse has been reduced to $2 \%$ compared to a Poisson-distributed source of the same intensity. The indistinguishability of emitted single photons from one of our devices has been tested through a Hong-Ou-Mandel-type two-photon interference experiment; consecutive photons emitted from such a source have been largely indistinguishable, with a mean wave-packet overlap as large as 0.81 . We have also designed and demonstrated two-dimensional photonic crystal GaAs cavities containing InAs quantum dots that exhibit much higher quality factors together with much smaller mode volumes than microposts, and therefore present an ideal platform for construction of single photon sources of even higher quality.
\end{abstract}

Keywords: Single photon sources, quantum dots, photonic crystals, quantum information science

\section{INTRODUCTION}

Generation of single photons at a well defined timing or clock is crucial for practical implementation of quantum key distribution (QKD), ${ }^{1}$ as well as for quantum computation ${ }^{2}$ and networking based on photonic qubits. ${ }^{3,4}$ Single-photon sources have recently been demonstrated using a variety of devices, including molecules, ${ }^{5-7}$ mesoscopic quantum wells, ${ }^{8}$ colour centers,${ }^{9}$ trapped ions, ${ }^{10}$ and semiconductor quantum dots. ${ }^{11-15}$ These sources rarely emit two or more photons in the same pulse, compared to a Poisson-distributed source of the same intensity. Three different criteria are taken into account when evaluating the quality of a single-photon source: high efficiency, small multi-photon probability (measured by the second-order coherence function $g^{(2)}(0)$ ), and quantum indistinguishability. For example, high efficiency and small $g^{(2)}(0)$ are required, but quantum indistinguishability is not necessary for BB84 QKD. ${ }^{16}$ On the other hand, for almost all other applications in the field of quantum information, including linear-optical quantum computation, ${ }^{17}$ we need photons that are indistinguishable and thus produce multi-photon interference. For a source based on a single quantum emitter, the emitter must therefore be excited in a rapid or deterministic way, and interact little with its surrounding environment.

One of the popular approaches to generation of single photons is based on a pulsed excitation of a semiconductor quantum dot (QD) combined with spectral filtering. ${ }^{11-15}$ Semiconductor quantum dots (QDs) are nano-scale inclusions of a low-bandgap semiconductor inside a semiconductor with a larger bandgap. The bandgap difference acts as a potential barrier for carriers, confining them inside the dot. Moreover, the dots are small enough that the confined carriers can only occupy discrete energy levels, and the transitions between such levels in the conduction band and the valence band involve the absorption or emission of photons at near-optical frequencies. Quantum dots can be formed spontaneously during epitaxial growth of lattice-mismatched materials, and such dots are called self-assembled. ${ }^{18}$ For example, when InAs is deposited on GaAs, a strained planar layer, known as a wetting layer, initially forms. The strain energy that builds up in this layer is eventually partially relieved by the formation of nanometer-scale islands on the surface, which can subsequently be covered with a capping layer of GaAs. InAs/GaAs quantum dots used in our experiments were self-assembled during molecular beam

Further author information: send correspondence to Jelena Vučković, E-mail: jela@stanford.edu, Telephone: 1650 725 2288, WWW: http://www.stanford.edu/group/nqp. 
epitaxy (MBE), under conditions that give relatively sparse dots, with a surface density of $11-75 \mu \mathrm{m}^{-2}$. Fig. 1 shows an atomic-force microscope image of dots similar to those used in our experiments, except for the absence of a GaAs capping layer.

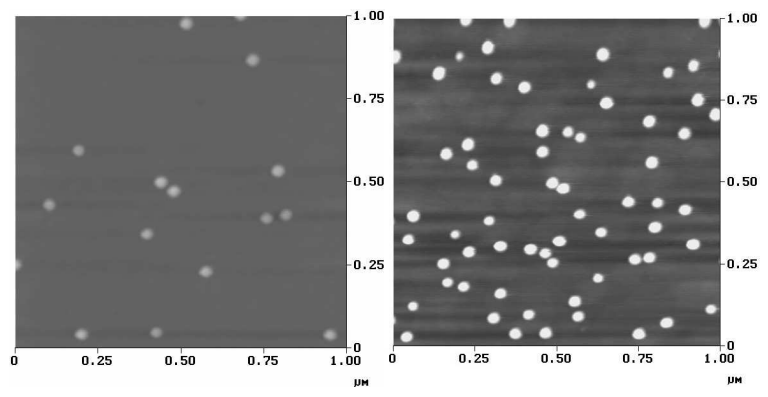

Figure 1. Atomic Force Microscope images of quantum dot arrays grown by Molecular Beam Epitaxy. The size and density of dots can be controlled by controlling the growth rate, the substrate temperature, the ratio of As to In impinging on the surface, and the amount of material deposited.

Although a single quantum dot by itself can be used to generate single photons, ${ }^{12}$ the efficiency of such a system is poor, as the majority of emitted photons are lost in the substrate. Moreover, emitted photons are unlikely to be indistinguishable, with coherence lengths shorter than the radiative limit (Fourier transform limit), and the single-photon generation rate is low, as determined by the long excitonic lifetime. Microcavities can help in correcting all of these deficiencies by employing cavity quantum electrodynamics (cavity QED) in the weak or strong coupling regimes. The weak coupling regime refers to the reduction of the radiative lifetime of an emitter on resonance with the cavity (i.e., the enhancement the spontaneous emission rate), ${ }^{11,15,19-21}$ while the strong coupling refers to the regime when the losses of the system (described by the cavity field decay rate $\kappa$, equal to $\omega / 2 Q$, and the excitonic dipole decay rate $\gamma$ ) are much smaller than the emitter-cavity field coupling parameter coupling parameter $g$. In the strong coupling regime, the reversible spontaneous emission, i.e., Rabi oscillation occurs ${ }^{22}$; this regime is not only of academic interest, but it can also be used in construction of sources of Fourier-transform limited single photons ${ }^{23}$ and components of quantum networks. ${ }^{24}$ Advantageous feature of solid-state microcavities is that a single narrow-linewidth emitter (quantum dot) can be embedded in them during the growth process, enabling cavity field interaction with an "artificial atom". Since the emitter-cavity field coupling paramneter $g$ is inversely proportional to the square root of the cavity mode volume $V$, and the cavity field decay rate is inversely proportional to the cavity Q-factor, this implies that the observation of cavity QED phenomena in semiconductor systems relies on the construction of optical microcavities with high quality factors $(Q)$ and small mode volumes $(V)$. In this article, we demonstrate our work on the quantum dot single photon sources based on two different types of cavities: DBR microposts and two dimensional photonic crystals of finite depths.

\section{QUANTUM DOT-MICROPOST SINGLE-PHOTON SOURCES}

We first describe experiments with an InAs quantum dot embedded in the middle of a GaAs spacer in a distributed Bragg reflector (DBR) micropost microcavity. The GaAs spacer is approximately one optical wavelength thick $(274 \mathrm{~nm})$, and sandwiched between twelve DBR mirror pairs on top, and thirty DBR mirror pairs on bottom. Each DBR pair consists of a $68.6 \mathrm{~nm}$ thick GaAs and a $81.4 \mathrm{~nm}$ thick AlAs layer (both layers are approximately a quarter-wavelength thick). The micropost structures (shown in Fig. 2) were constructed by a combination of molecular-beam epitaxy (MBE) and chemically assisted ion beam etching (CAIBE). MBE was used to grow a wafer consisting of DBR mirrors and a GaAs spacer with embedded self-assembled QDs. Microposts with diameters ranging from $0.3 \mu \mathrm{m}$ to $5 \mu \mathrm{m}$ and heights of $5 \mu \mathrm{m}$ were fabricated in a random distribution by CAIBE, with $\mathrm{Ar}^{+}$ions and $\mathrm{Cl}_{2}$ gas, and using sapphire $\left(\mathrm{Al}_{2} \mathrm{O}_{3}\right)$ dust particles as etch masks. Sapphire was chosen as a mask because of its chemical stability and hardness, which enabled larger etch depths than other mask-materials. Unfortunately, these same properties impede its removal from the tops of the structures, without endangering the 
microposts. ${ }^{25}$ The presence of sapphire on top of the structures decreases their quality factors and consequently reduces outcoupling efficiencies. This can be confirmed theoretically by the Finite-Difference Time-Domain (FDTD) calculation of the $Q$-factor of the fundamental $\mathrm{HE}_{11}$ mode (whose field pattern is also shown in Fig. 2 ) in a micropost with and without sapphire on top. The FDTD unit cell size used in this case is $5 \mathrm{~nm}$. We assume that the DBR layers of the simulated post have the same parameters as in the experimentally studied structures, that the post has perfectly straight walls, diameter of $0.5 \mu \mathrm{m}$, and that refractive indices of GaAs, AlAs, and $\mathrm{Al}_{2} \mathrm{O}_{3}$ are 3.5, 2.9, and 1.75, respectively. Without sapphire, the $Q$-factor of the $\mathrm{HE}_{11}$ mode is 2600 and its wavelength is $882 \mathrm{~nm}$; with a $0.5 \mu \mathrm{m}$ thick sapphire disk on top of the post (with diameter equal to that of the post), the $Q$-factor drops to 1400 , while the mode wavelength remains unchanged. Due to the irregular shapes of the fabricated posts, the $\mathrm{HE}_{11}$ mode is typically polarization-nondegenerate, and many microposts have only one or two QDs on resonance with this fundamental mode. An additional factor contributing to the degradation of the Q-factor of the fundamental mode is the imperfect wall profile, i.e., the undercut that can be seen in Fig. 2, as well as the insufficient etch depth. ${ }^{26}$ We optimized our fabrication method in such a way to minimize both of these effects.

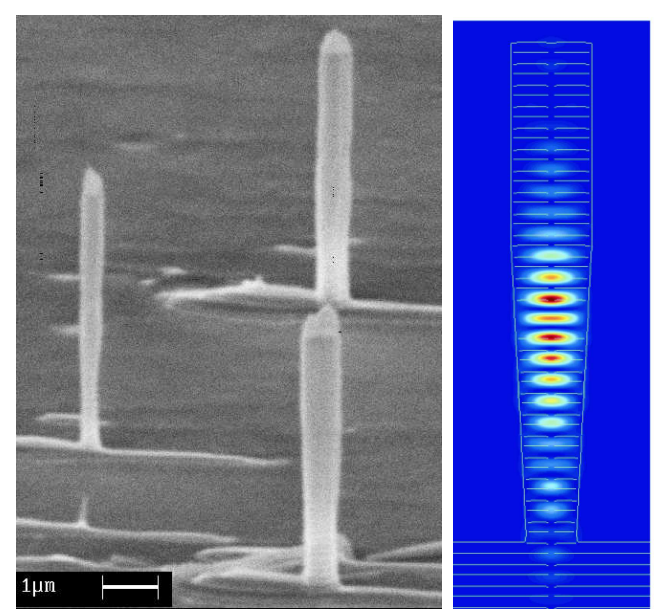

Figure 2. Left: Scanning electron micrograph showing a fabricated array of microposts. Right: Electric field amplitude for the fundamental mode in a micropost with realistic wall profile.
The single quantum dots embedded in such microposts were probed by photoluminescence (PL), and Fig. 3 shows the experimental apparatus used. The sample is placed in a liquid $\mathrm{He}$ cryostat, and posts containing quantum dots are excited from a steep angle by Ti:sapphire laser pulses, 3 ps in duration, with a $76 \mathrm{MHz}$ repetition rate. The emission from the dot is collected normal to the sample surface with an aspheric lens that has a numerical aperature of 0.5 , and is focused onto a pinhole that effectively selects a $5 \mu \mathrm{m}$ region of the sample for collection. The collected emission is then directed towards a streak camera preceded by a spectrometer, for time-resolved photoluminescence measurements. The spectral resolution of the system is $0.1 \mathrm{~nm}$, together with the time resolution of $25 \mathrm{ps}$. For photon correlation measurements, the collected emission is first spectrally filtered and then directed towards a Hanbury Brown and Twiss-type (HBT) setup. In the HBT setup, photon counters are placed at both outputs of a non-polarizing beamsplitter for detection. The electronic signals from the counters are sent to a time-to-amplitude converter followed by a multi-channel analyzer computer card, which generates a histogram of the relative delay time $\tau=t_{2}-t_{1}$ between a photon detection at one counter $\left(t_{2}\right)$ and the other $\left(t_{1}\right)$.

The excitation frequency of the Ti:sapphire laser is either above the bandgap of the GaAs surrounding InAs QDs, or is resonant with higher-level confined states of a QD. The former type of the excitation is called aboveband excitation, and the latter is referred to as resonant excitation. In above-band excitation, electron-hole pairs are created in the surrounding semiconductor (GaAs) matrix, after which they diffuse towards the dot, where they relax to the lowest confined states. The created carriers recombine in a radiative cascade, leading to the generation of several photons for each laser pulse. All of these photons have slightly different frequencies, resulting from the Coulomb interaction among carriers. The last emitted photon for each pulse has a unique frequency, and can be spectrally isolated. With resonant excitation, electron-hole pairs are created within the dot, and the favored absorption of a single electron-hole pair and formation of one-exciton state is expected. This is illustrated in Fig. 4. With continuous-wave (CW) excitation above the GaAs bandgap, the emission spectrum displays several lines. When the laser is tuned to an absorption resonance of a higher-level confined state of a QD, thus creating excitons directly inside the dot, many emission peaks almost disappear, and we therefore believe that they represent emission from charged states of the dot.

As described previously, the last photon emitted for each excitation pulse corresponds to a single-exciton state and has a unique frequency corresponding to peak at $932 \mathrm{~nm}$ in Fig. 4. If this photon is spectrally filtered at the 


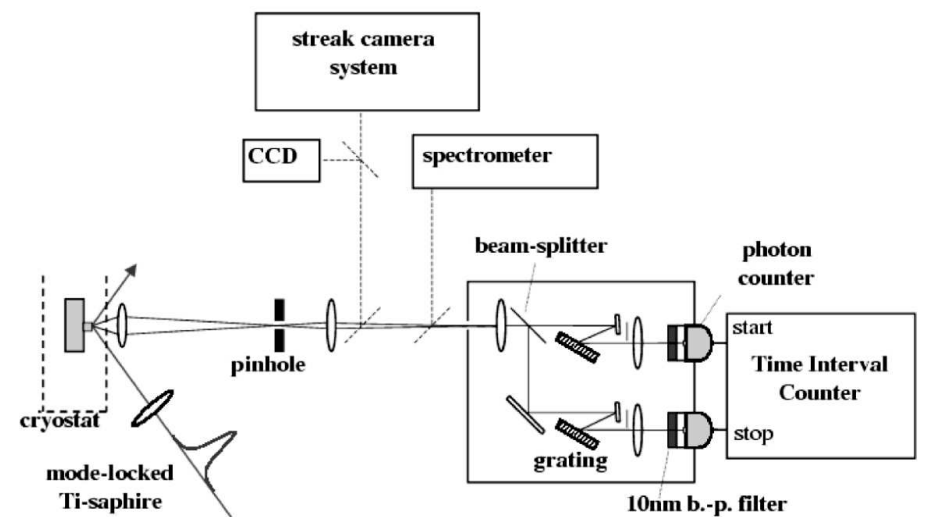

Figure 3. Experimental setup used to measure luminescence from single quantum dots.
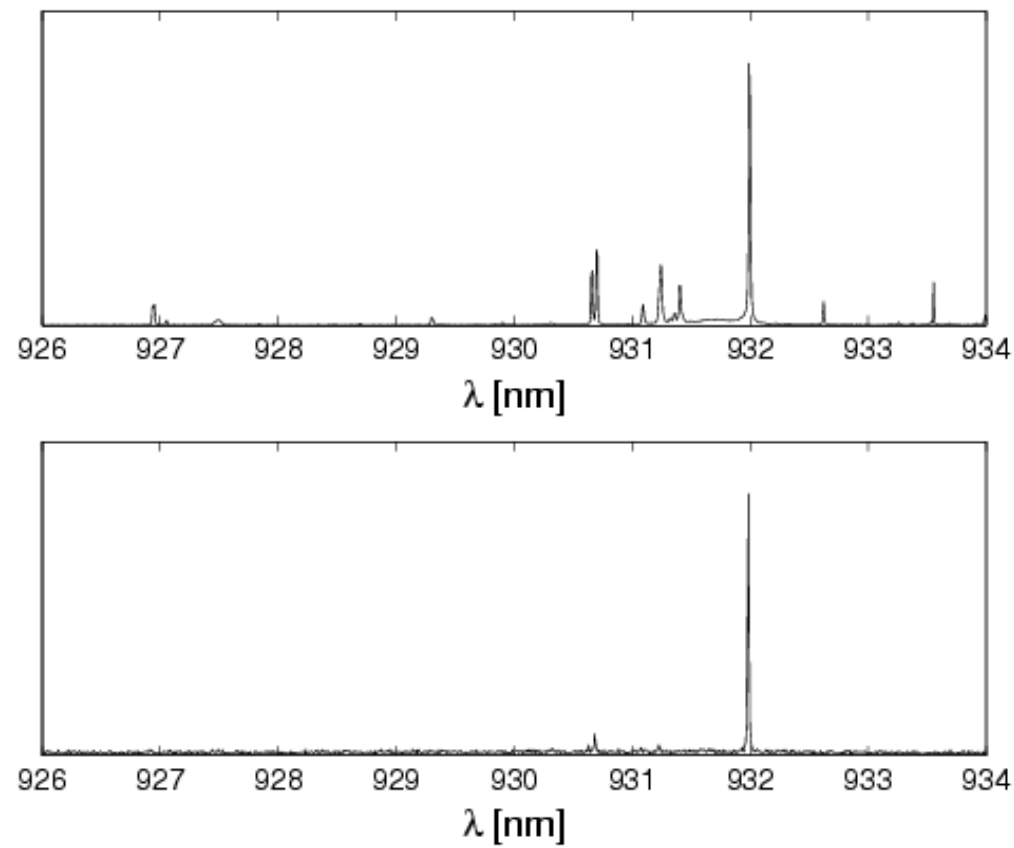

Figure 4. Photoluminescence from a single InAs/GaAs quantum dot under above-band excitation (top) and resonant excitation (bottom). With resonant excitation, the favored absorption of a single electron-hole pair and formation of one-exciton state is expected.

output of a quantum dot source, then a single-photon emission on demand is achieved, since only one photon is present at the output of the source for each laser pulse. ${ }^{12}$ Besides narrow-bandwidth spectral filtering, it is also necessary that the laser excitation pulses be much shorter than the lifetimes of carriers in the dot, thereby suppressing the re-excitation of the dot in the same pulse. A pulse stream with reduced multi-photon probability compared to the Poissonian case is said to be antibunched, and can only be described quantum mechanically. Mathematically, such non-classical photon statistics can be described using the second-order coherence function $g^{(2)}(\tau)$. To characterize our single-photon source, we employ the previously described HBT setup. The HBT setup can be used to measure the photon correlation histogram which corresponds to the second-order coherence function $\left(g^{(2)}(\tau)\right)$ in the limit of low collection and detection efficiency. ${ }^{27}$ Of special interest is the central peak in $g^{(2)}(\tau)$ at $\tau=0$, which gives an upper bound on the probability to generate two or more photons in a given 
pulse $^{12}$ :

$$
P(n \geq 2) \leq \frac{1}{2} \bar{n}^{2} g^{(2)}(0),
$$

where $\bar{n}$ is the mean photon number per pulse.

A single quantum dot by itself can be used to generate single photons, but the efficiency of such a system is poor, as the majority of emitted photons are lost in the substrate. In addition, the radiative lifetime can be as long as $1 \mathrm{~ns}$, which is greater than the dephasing time estimated to be $\sim 0.9 \mathrm{~ns}$ at $4 \mathrm{~K} .^{28}$ Emitted photons are unlikely to be indistinguishable, with coherence lengths shorter than the radiative limit (Fourier transform limit). Finally, the single-photon generation rate is low, as determined by the long excitonic lifetime. Microcavities can help in correcting all of these deficiencies. ${ }^{11,15,19-21}$ The radiative lifetime of an emitter on resonance with the cavity can be decreased significantly below the dephasing time, bringing the emitted photon pulses closer to the Fourier transform limit. Moreover, the spontaneous emission rate can be enhanced, and a large fraction of spontaneously emitted photons can be coupled into a single cavity mode, thereby increasing the outcoupling efficiency.

We performed lifetime and $g^{(2)}$ measurements on a QD chosen for its bright emission under resonant excitation. Tuning of the sample temperature was used to tune the emission wavelength relative to the cavity resonance. ${ }^{29}$ In the studied temperature range, the shift of the cavity resonance with temperature (due to refractive index change) is much slower than the shift in the excitonic emission wavelength, which is attributed to the changes in the band gaps of InAs and GaAs with temperature. ${ }^{29}$ This can be seen in Fig. 5, where streak camera images taken at $10 \mathrm{~K}$ and $30 \mathrm{~K}$ for the same resonantly excited dot are shown. Clearly, the emission wavelength increases with temperature; as a result of this shift, the radiative lifetime also changes. This particular dot (presented in Figs. 5 and 6) is almost exactly on resonance with the cavity at low temperature, so by heating the sample and increasing the QD emission wavelength, one also increases the detuning from the cavity resonance and the radiative lifetime. The opposite process is observed if the QD emission wavelength is initially smaller than the cavity resonance.
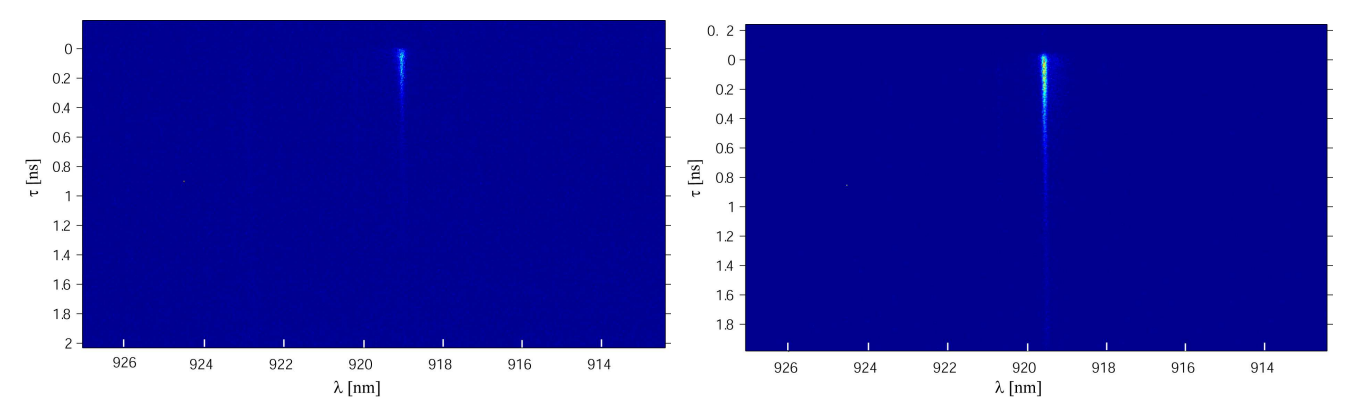

Figure 5. Streak camera images of a quantum dot emission taken at the temperatures of 10K (left) and 30K (right).

In addition to a good correspondence between the cavity resonance linewidth and the linewidth of Lorentzian describing decay rate of the emission line as a function of detuning from the cavity resonance (see Fig. 6 top-left and top-right), these processes indicate that cavity QED has a dominant effect on the radiative lifetime. Fig. 6 (bottom) shows the time-resolved photoluminescence of an emission line on- and off- resonance with the cavity mode. The decay lifetime differs by a factor of five for these two cases. The decay rate for this emission line as a function of the absolute value of its detuning from the cavity resonance $\left(\left|\lambda_{Q D}-\lambda_{c}\right|\right)$ is plotted with circles in the top-right plot of Fig. 6. The solid line corresponds to the Lorentzian fit to the experimental data, ${ }^{19}$ and a good match is observed between our experiment and the theoretically predicted behavior. The fitting parameters are the linewidth, maximum and minimum of the Lorentzian, while it is assumed that its central wavelength is equal to $\lambda_{c}$, the cavity resonance wavelength. $\lambda_{c}$ and the quality factor $(Q=1270)$ are determined from the photoluminescence intensity taken at high pump powers (see the top-left plot of Fig. 6). The cavity resonance wavelength red-shifts by roughly $0.3 \mathrm{~nm}$ with increasing temperature in the studied range, and this 
shift is included in plotting the data. Up to fivefold spontaneous emission rate enhancement (Purcell factor $F$ ) is observed for the dot coupled to a cavity, as opposed to the dot off of resonance (top-right plot of Figure 6).
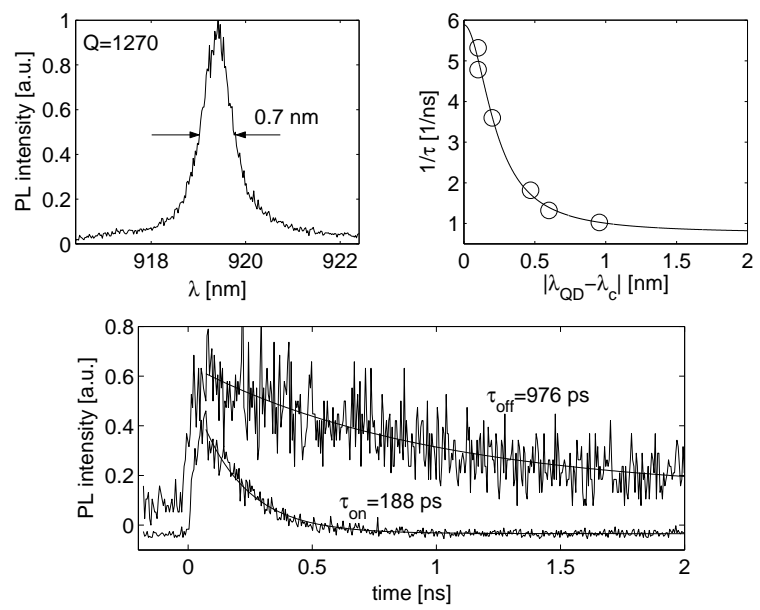

Figure 6. Top-left: Background emission filtered by the cavity. Top-right: Decay rate of the emission line as a function of the absolute value of its detuning from the cavity resonance $\left(\left|\lambda_{Q D}-\lambda_{c}\right|\right)$. The dot emission wavelength was tuned by changing the sample temperature within the $6 \mathrm{~K}-40 \mathrm{~K}$ range. Bottom: time-dependent photoluminescence from the emission line on-resonance with the cavity, as opposed to this same emission line off-resonance.

The theoretical limit of the $Q$-factor of the studied microposts is 4000 , which is the value calculated for a planar cavity (before etching) without any absorption losses and inaccuracies in the growth of DBR layers. The maximum $Q$-factor of a micropost with a finite diameter has to be below this limit, due to the additional loss mechanisms in the transverse directions ${ }^{30}$ and the presence of sapphire dust particles, as discussed above. According to the detailed theoretical treatment of the QD micropost device, ${ }^{26,30}$ the cavity $Q$-factor and Purcell factor can be much larger $(Q \sim 10000$ and $F \sim 100)$ for optimized cavity designs with 15 and 30 DBR pairs on top and bottom, respectively, perfectly straight cavity walls, and a QD located at the cavity center.

In addition to the small multi-photon probability, a strong Purcell effect is important in a single-photon source for improving the photon outcoupling efficiency and the single-photon generation rate, and for bringing the emitted photon pulses closer to the Fourier transform limit. In this section, we describe experimental demonstration of a single-photon source based on a quantum dot in a micropost microcavity that exhibits a large Purcell factor together with a small multi-photon probability: for a quantum dot on resonance with the cavity, the spontaneous emission rate is increased by a factor of five, while the probability to emit two or more photons in the same pulse is reduced to $2 \%$ compared to a Poisson-distributed source of the same intensity.

A photon correlation histogram was measured for the same quantum dot from Figure 6 on resonance with the cavity, and with an excitonic radiative lifetime below 200 ps. The collected emission was first spectrally filtered with a bandwidth of $0.1 \mathrm{~nm}$, and then directed towards the previously described Hanbury Brown and Twiss-type (HBT) setup; the measured histogram is shown in Fig. 7. The distance between peaks is 13 ns, corresponding to the repetition period of pulses from the Ti:sapphire laser. The decrease in the height of the side peaks as $|\tau|$ increases indicates the dot blinking behavior generally observed under resonant excitation, and can be approximated with a double-sided exponential. ${ }^{12}$ The vanishing central peak (at $\tau=0$ ) indicates a strong antibunching and a large suppression of multi-photon pulses. The probability of generating two or more photons for the same laser pulse compared to a Poisson-distributed source of the same intensity $\left(g^{(2)}(0)\right)$ is estimated from the ratio of the areas of the central peak and the peaks at $|\tau| \rightarrow \infty$. Each area is calculated by integrating all the counts within the integration window centered at the peak and without subtracting any background counts. The peak at $|\tau| \rightarrow \infty$ is estimated from the decaying exponential fit to the heights of the side peaks. $g^{(2)}(0)$ is estimated to be equal to $2 \%$ for an integration window of $4 \mathrm{~ns}$. The integration window is chosen so that the contribution of the peak tails outside it to the peak area can be neglected (it is below 1\%). The width of the 


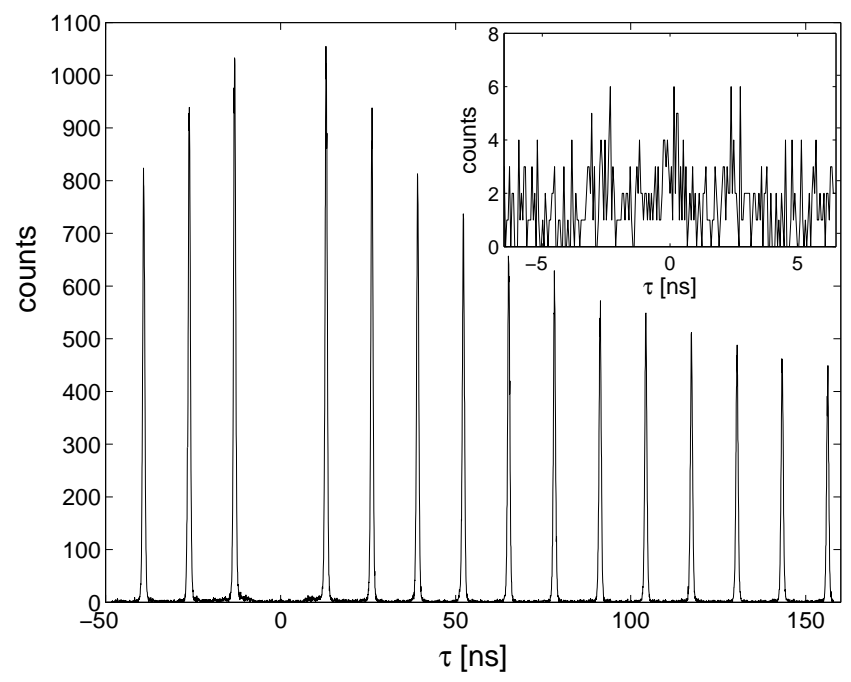

Figure 7. Photon correlation histogram for a QD on resonance with the cavity (the lifetime of this dot is shown in Fig. 6 ), under pulsed, resonant excitation. The inset depicts the magnified central portion (from $\tau=-6.5 \mathrm{~ns}$ to $6.5 \mathrm{~ns}$ ) of the histogram. The missing central peak (at $\tau=0$ ) indicates a large suppression of multi-photon pulses.

histogram peaks is determined by the photon counter timing resolution ( $0.3 \mathrm{~ns})$ and the excitonic lifetime (below $0.2 \mathrm{~ns}){ }^{12}$ Owing to the strong Purcell effect, this small multiphoton probability should be preserved even for a repetition period much smaller than $13 \mathrm{~ns}$ (e.g., $2 \mathrm{~ns}$ ). If the integration window is reduced to $1 \mathrm{~ns}, g^{(2)}(0)$ drops to $1 \%$. Depending on the application of the photon-source, a different definition of the two-photon probability may be necessary. For example, in an interference experiment using two consecutive photons emitted from a dot, the relevant parameter is the probability to emit two photons in the same pulse, as opposed to the probability to emit one photon in each of two consecutive pulses. ${ }^{20}$ This parameter is calculated from the ratio of the area of the central peak to the area of the nearest side peak, and is equal to $0.9 \%$ for the integration window of 4 ns. The difference between this parameter and $g^{(2)}(0)$ is a result of the blinking behavior of the dot.

As we stated previously, for most applications in the field of quantum information we need photons that are quantum-mechanically indistinguishable and can thus produce multi-photon interference. We have tested the indistinguishability of photons emitted by a semiconductor quantum dot in a micropost microcavity through a Hong-Ou-Mandel-type two-photon interference experiment, ${ }^{31,32}$ and found that consecutive photons have a mean wave-packet overlap as large as 0.81 , making this source useful for a variety applications in quantum optics and quantum information. For example, we have employed such single-photon sources to generate polarizationentangled photons by post-selection ${ }^{33}$ and for single-mode quantum teleportation. ${ }^{34}$

\section{QUANTUM DOT-PHOTONIC CRYSTAL CAVITY SINGLE PHOTON SOURCES}

We have recently proposed the two-dimensional photonic crystal cavity of finite depth shown in Fig. $8{ }^{35}$ which can enable much higher $Q$ factors together with much smaller mode volumes $V$ than the microposts employed in the previously described experiments; this in turn, can be employed to improve the properties of the single photon sources. The cavity shown in Fig. 8 is an extension of the design we proposed for cavity QED with neutral atoms, ${ }^{36}$ but with the electric field maximum in the high-index region at the cavity center, to facilitate its alignment to a QD and to prevent nonradiative surface recombination. Theoretically, Q-factors above 40000 are possible together with mode volumes of around $0.5(\lambda / n)^{3}$. Experimentally, we have been able to measure Q-factors between 2500 and 4300 for such cavities (see Fig. 9), which is already leading to an order of magnitude higher $Q / V$ ratio than in our best microposts. By employing the FDTD method, we have confirmed that the degradation of $Q$ relative to the theoretical prediction is resulting mainly from the fabrication imperfection (the simulated $Q$ of the fabricated cavities is around 5000). However, the experimentally observed cavity parameters 


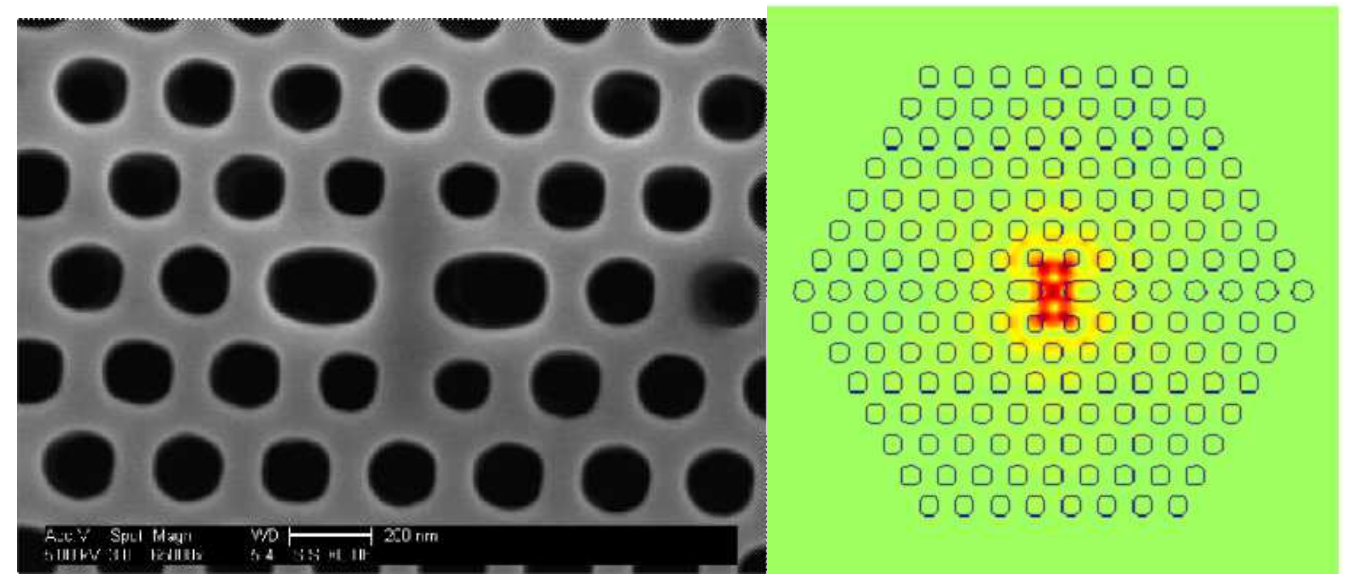

Figure 8. Photonic crystal cavity fabricated in GaAs containing InAs QDs, for operation around 950nm. Such cavities exhibit mode volumes of around $0.5(\lambda / n)^{3}$ for the dipole mode whose electric field pattern is shown in the figure on the right, and we have measured Q-factors of up to 4300.

are sufficient to enable the onset of the strong coupling regime for a single self-assembled InAs/GaAs QD embedded inside such a cavity ( $g$ is of the order of $\kappa$ and much larger than $\gamma$ ), which can be employed in construction of high-visibility single photon sources. ${ }^{23}$

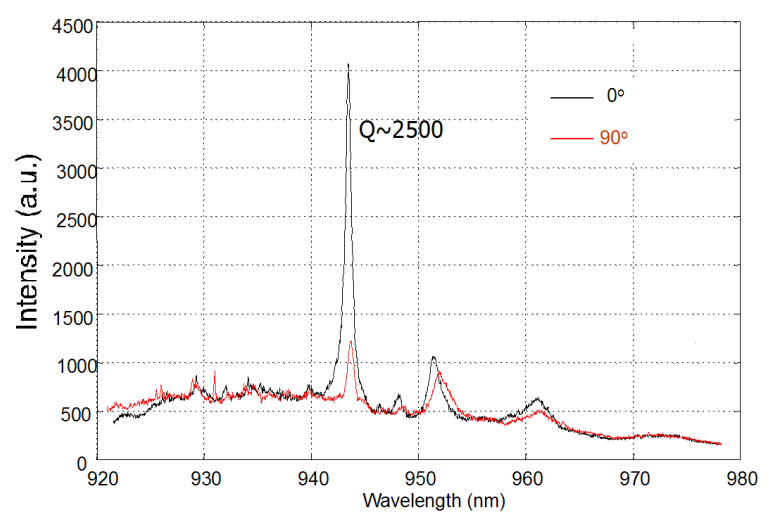

Figure 9. Spectrum of the cavity such as the one shown in Fig. 8. Polarization sensitivity of the spectrum implies that the main peak corresponds to the dipole mode, which is primarily linearly polarized.

We have measured Q-factors of these cavities in the same way as those of microposts, i.e., by measuring the QD photoluminescence at high excitation powers. Figure 9 shows the photoluminescence spectrum of such a cavity with $\mathrm{Q}=2500$. Polarization sensitivity of the spectrum implies that the main peak corresponds to the dipole mode, which is primarily linearly polarized. The power series showing the transition between the QD and the cavity spectrum is shown in Fig. 10. The cavity resonance linewidth narrowing at high-excitation powers is not present, implying that the measured Q-factors cannot be overestimated because of the onset of the stimulated emission and should be close to the cold-cavity values. In addition, these spectra also confirm that the quantum dot density is low in these structures and that the spectroscopy of a single quantum emitter can be performed (as can be additionally proved by measuring $g^{(2)}(\tau)$ and observing antibunching). Our experimental work on the 
study of the cavity QED with a single emitter in such a system and single-photon generation on demand will be reported in our forthcoming publication.

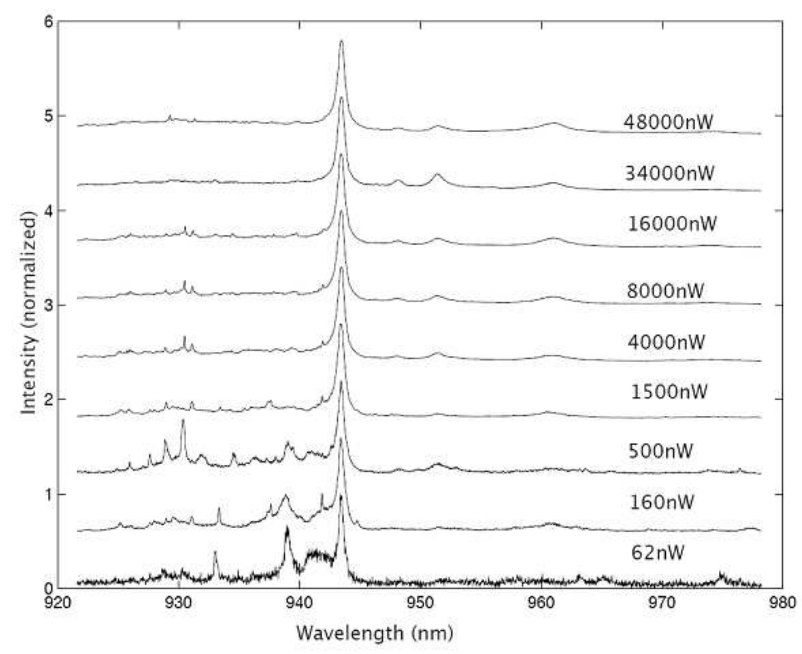

Figure 10. Power series for the photoluminescence from a photonic crystal cavity containing QDs, showing a transition between the quantum dot spectrum at low excitation powers and the cavity spectrum at high excitation powers.

\section{CONCLUSIONS AND FUTURE PROSPECTS}

Single photons on demand can be generated by combining pulsed excitation of a single semiconductor quantum dot and spectral filtering, but properties of such a source can be significantly improved by embedding a quantum dot inside an optical microcavity with a high quality factor and small mode volume. The radiative lifetime of an emitter on resonance with the cavity can be decreased significantly below the dephasing time, bringing the emitted photon pulses closer to the Fourier transform limit. Moreover, a large fraction of spontaneously emitted photons can be coupled into a single cavity mode, thereby increasing the outcoupling efficiency. Finally, the single-photon generation rate increases with a cavity-induced reduction in the excitonic lifetime. We have demonstrated a single-photon source based on a quantum dot in a micropost microcavity that exhibits a large Purcell factor together with a small multi-photon probability. For a quantum dot on resonance with the cavity, the spontaneous emission rate is increased by a factor of five (Purcell factor $F=5$ ), while the probability to emit two or more photons in the same pulse is reduced to $2 \%$ compared to a Poisson-distributed source of the same intensity $\left(g^{(2)}(0)=2 \%\right)$. The indistinguishability of emitted single photons from one of our devices was tested through a Hong-Ou-Mandel-type two-photon interference experiment. We found that consecutive photons are largely indistinguishable, with a mean wave-packet overlap as large as 0.81 , making this source useful in a variety of experiments in the field of quantum information. We have also designed, fabricated and tested planar GaAs photonic crystal microcavities with embedded low-density of self-assembled InAs/GaAs quantum dots, which exhibit much higher $Q$-factors together with much smaller mode volumes than the previously employed microposts; in addition, they have a maximum field intensity located in the high-refractive index region, which can be used for enhancing interaction between quantum dots and cavity field. Cavity QED in the strong coupling regime with a single quantum dot is possible in such a cavity, opening a possibility for novel solid-state quantum optical devices, including sources of Fourier-transform limited single photons or components of quantum networks.

\section{ACKNOWLEDGMENTS}

This work has been supported by MURI Center for Photonic Quantum Information Systems (ARO/ARDA Program DAAD19-03-1-0199), by the Quantum Entanglement Project ICORP-JST, and by the NSF Grant ECS0424080. The authors would like to thank A. Scherer (Caltech) for providing access to CAIBE for micropost etching, and B. Zhang for AFM images of quantum dot arrays. 


\section{REFERENCES}

1. C. H. Bennet and G. Brassard, Proceedings of the IEEE International Conference on Computers, Systems and Signal Processing, IEEE, Bangalore, India, 1984.

2. E. Knill, R. Laflamme, and G. J. Milburn, "A scheme for efficient quantum computation with linear optics," Nature 409, pp. 46-52, 2001.

3. J. Cirac, P. Zoller, H. Kimble, and H. Mabuchi, "Quantum state transfer and entanglement distribution among distant nodes in a quantum network," Physical Review Letters 78, p. 3221, 1997.

4. L. Duan, M. Lukin, J. Cirac, and P. Zoller, "Long-distance quantum communication with atomic ensembles and linear optics," Nature 414, pp. 413-418, 2001.

5. F. D. Martini, G. D. Giuseppe, and M. Morocco, "Single-mode generation of quantum photon states by excited single molecules in a microcavity trap," Physical Review Letters 76, pp. 900-903, 1996.

6. C. Brunel, B. Lounis, P. Tamarat, and M. Orrit, "Triggered source of single photons based on controlled single molecule fluorescence," Physical Review Letters 83, pp. 2722-2725, 1999.

7. B. Lounis and W. E. Moerner, "Single photons on demand from a single molecule at room temperature," Nature 407, pp. 491-493, 2000.

8. J. Kim, O. Benson, H. Kan, and Y. Yamamoto, "A single-photon turnstile device," Nature 297, pp. 500-503, 1999.

9. A. Beveratos, S. Kuhn, R. Brouri, T. Gacoin, J. Poizat, and P. Grangier, "Room temperature stable singlephoton source," Eur. Phys. J. D 18, pp. 191-196, 2002.

10. A. Kuhn, M. Hennrich, and G. Rempe, "Deterministic single-photon source for distributed quantum networking," Physical review Letters 89, p. 067901, 2002.

11. P. Michler, A. Kiraz, C. Becher, W. V. Schoenfeld, P. M. Petroff, L. Zhang, E. Hu, and A. Imamoğlu, "A quantum dot single-photon turnstile device," Science 290, pp. 2282-2285, 2000.

12. C. Santori, M. Pelton, G. Solomon, Y. Dale, and Y. Yamamoto, "Triggered single photons from a quantum dot," Physical Review Letters 86(8), pp. 1502-1505, 2001.

13. V. Zwiller, H. Blom, P. Jonsson, N. Panev, S. Jeppesen, T. Tsegaye, E. Goobar, M. E. Pistol, L. Samuelson, and G. Bjork, "Single quantum dots emit single photons at a time: antibunching experiments," Applied Physics Letters 78, pp. 2476-2478, 2001.

14. Z. Yuan, B. E. Kardynal, R. M. Stevenson, A. J. Shields, C. J. Lobo, K. Cooper, N. S. Beattie, D. A. Ritchie, and M. Pepper, "Electrically driven single-photon source," Science 295, pp. 102-105, 2002.

15. E. Moreau, I. Robert, J. M. Gérard, I. Abram, L. Manin, and V. Thierry-Mieg, "Single-mode solid-state single photon source based on isolated quantum dots in pillar microcavities," Applied Physics Letters 79(18), pp. 2865-2867, 2001.

16. E. Waks, C. Santori, and Y. Yamamoto, "Security aspects of quantum key distribution with sub-Poissonian light," Physical Review A 66, p. 042315, Oct. 2002.

17. E. Knill, R. Laflamme, and G. J. Milburn, "A scheme for efficient quantum computation with linear optics," Nature 409, pp. 46-52, 2001.

18. M. Tabuchi, S. Noda, and A. Sasaki, Science and Technology of Mesoscopic Structures, p. 375. SpringerVerlag, Tokyo, 1992.

19. J. M. Gérard, B. Sermage, B. Gayral, B. Legrand, E. Costard, and V. Thierry-Mieg, "Enhanced spontaneous emission by quantum boxes in a monolithic optical microcavity," Physical Review Letters 81, pp. 1110-1113, 1998.

20. C. Santori, D. Fattal, J. Vučković, G. Solomon, and Y. Yamamoto, "Indistinguishable photons from a single-photon device," Nature 419, pp. 594-597, Oct. 2002.

21. J. Vučković, D. Fattal, C. Santori, G. Solomon, and Y. Yamamoto, "Enhanced single photon emission from a quantum dot in a micropost microcavity," Applied Physics Letters 82, pp. 3596-3598, May 2003.

22. H. J. Kimble, in Cavity Quantum Electrodynamics, edited by P. Berman, Academic Press, San Diego, 1994.

23. A. Kiraz, M. Atature, and A. Imamoğlu, "Quantum dot single-photon sources: prospects for applications in linear optical quantum information processing," Physical Review A 69, p. 032305, 2004. 
24. H. Mabuchi, M. Armen, B. Lev, M. Lončar, J. Vučković, H. J. Kimble, J. Preskill, M. Roukes, and A. Scherer, "Quantum networks based on cavity QED," Quantum Information and Computation (special issue on Implementation of Quantum Computation) 1, pp. 7-12, 2001.

25. P. Levy, M. Bianconi, and L. Correra, "Wet etching of $\mathrm{Al}_{2} \mathrm{O}_{3}$ for selective patterning of microstructures using $\mathrm{Ar}^{+}$ion implantation and $\mathrm{H}_{3} \mathrm{PO}_{4}$," Journal of the Electrochemical Society 145, p. 344, 1998.

26. M. Pelton, J. Vučković, G. S. Solomon, A. Scherer, and Y. Yamamoto, "Three-dimensionally confined modes in micropost microcavities: Quality factors and Purcell factors," IEEE Journal of Quantum Electronics 38(2), pp. 170-177, 2002.

27. S. Reynaud, "Resonance fluorescence - the dressed atom approach," Annales de Physique 8, pp. 315-370, 1983.

28. M. Bayer and A. Forchel, "Temperature dependence of the exciton homogeneous linewidth in InGaAs/GaAs self-assembled quantunm dots," Physical Review B 65, p. 041308, 2002.

29. A. Kiraz, P. Michler, C. Becher, B. Gayral, A. Imamoğlu, L. Zhang, E. Hu, W. V. Schoenfeld, and P. M. Petroff, "Cavity-quantum electrodynamics using a single InAs quantum dot in a microdisk structure," Applied Physics Letters 78, pp. 3932-3934, 2001.

30. J. Vučković, M. Pelton, A. Scherer, and Y. Yamamoto, "Optimization of three-dimensional micropost microcavities for cavity quantum electrodynamics," Physical Review A 66, p. 023808, Aug. 2002.

31. H. Fearn and R. Loudon, "Theory of two-photon interference," Journal of the Optical Society of America $B$ 6, pp. 917-927, 1989.

32. C. K. Hong, Z. Y. Ou, and L. Mandel, "Measurement of subpicosecond time intervals between two photons by interference," Physical Review Letters 59, pp. 2044-2046, 1987.

33. D. Fattal, K. Innoue, J. Vuckovic, C. Santori, G. Solomon, and Y. Yamamoto, "Entanglement formation and violation of Bell's inequality with a semiconductor single photon source," Physical Review Letters 92, p. 037903, 2004.

34. D. Fattal, E. Diamanti, K. Innoue, and Y. Yamamoto, "Quantum Teleportation with a Quantum Dot Single-photon source," Physical Review Letters 92, p. 037904, 2004.

35. J. Vučković and Y. Yamamoto, "Photonic crystal microcavities for cavity quantum electrodynamics with a single quantum dot," Applied Physics Letters 82, pp. 2374-2376, Apr. 2003.

36. J. Vučković, M. Lončar, H. Mabuchi, and A. Scherer, "Design of photonic crystal microcavities for cavity QED," Physical Review E 65, p. 016608, 2002. 